\title{
Study of heat stress memory related to acquired thermo-tolerance in wheat
}

\author{
Mahmoud A. El-Rawy \\ Department of Genetics, Faculty of Agriculture, Assiut University, Assiut, Egypt \\ Corresponding author email: mabosuud2020@aun.edu.eg
}

Received on: 28/9/2020

Accepted on: 4/11/2020

\begin{abstract}
Wheat is one of the important plants frequently suffer by effects of heat stress. In the present study, heat priming for the first generation studied on heat tolerance of the successive generations in seedling and maturity stages under high temperature stress. Under high-temperature stress condition during seedling stage, the progeny of heat-primed (PH) genotypes shown significantly higher chlorophyll a and $\mathrm{b}$ content and percent tetrazolium chloride (\% TTC) reduction than the progeny of the non-heat primed $(\mathrm{NH})$ genotypes. At maturity stage, higher grain yield per plant was obtained for PH genotypes than $\mathrm{NH}$ genotypes under heat stress condition, which was mainly associated with the higher grain weight for $\mathrm{PH}$ than $\mathrm{NH}$ genotypes. The greater grain weight could be resulted from the higher percent cell membrane thermo-stability (\%CMS) in the $\mathrm{PH}$ genotypes than in the $\mathrm{NH}$ genotypes. Estimation of variance components and broad sense heritability were higher in $\mathrm{PH}$ genotypes than observed in NH genotypes for most studied traits at seedling and maturity stages. Based on morphophysiological traits, the highest genetic distance was recorded between G9 genotype of heat-primed plants and G9 genotype of non-primed plants, which gave highly significant differences between $\mathrm{PH}$ plants and $\mathrm{NH}$ plants for all studied traits. The heat priming for the parents may induce heat tolerance for offspring, and this might be an effective method to cope with severe heat stress during some the different growth stages in wheat.
\end{abstract}

KEYWORDS: Wheat, Heat stress, Epigenetics, Stress memory.

\section{INTRODUCTION}

The worldwide is used wheat as staple food. However, grain yield of wheat decreases largely under biotic and abiotic stresses. With climate change of the global, the occurrences of extreme weather events such as drought and heat stresses have significantly increased, in terms of frequency, duration and extent (Field et al. 2014). The optimum temperature of growth in wheat is around $21^{\circ} \mathrm{C}$ during reproductive growth (Porter and Gawith 1999). During grain filling, wheat plants are high sensitive to high temperature stress. Heat priming for the parent was studied on tolerance of the progeny of parents in post-anthesis under high temperature stress (Wang et al. 2016). Pre-exposure of plants to moderate stress may bring about "stress memory" that helps a fast protective response to next stress event (Boyko and Kovalchuk 2011). Stress memory has been defined as physiological, genetic and epigenetic changes under stress conditions, and it modifies responses to reoccurring stress in the same generation or in the next generation. (Boyko and Kovalchuk 2011). Stress memory in the same generation has been found to be correlated with increased tolerance to several abiotic or biotic stresses in many species of plants (Conrath et al. 2006). Molecular analysis displays that stress memory induced by pathogen attack (Conrath 2011), are associated with epigenetic variations (such as chromatin remodeling, DNA methylation, or small interfering RNAs), chromatin modifications, signaling proteins accumulation, and primary of metabolism alterations (Conrath 2011).

Hsieh et al. (2009) reported that a general mechanism supposed to restrict transmission of acquired epigenetic states between generations includes establishing of a default epigenetic status during gametogenesis and early phases of embryo development. Previous studies have appeared that midheat priming applied before anthesis effectively decreased the impacts of serious high temperature stress occurring during grain filling. This reduction was attributed to diminished damage by sub-cellular levels and oxidative stress at leaf, and thus a large maintenance of photosynthesis (Wang et al. 2011; 2014), and to enhanced carbohydrate remobilization from stems to grains (Wang et al. 2012). The primed 
heat treatment induces improving tolerance to stress reoccurring in the next generation (Rasmann et al. 2012). The researches in the future are needed to confirm the molecular mechanisms in which plant store information during stress exposure because biotic and abiotic stresses limit agricultural production and stress responses often lead to down-regulation of yield determining processes such as photosynthesis.

The current study aims to, (1): Impacts of the pre-exposure to heat stress on heat tolerance induced in the next generations. (2): Determine to the situation of several physiological and morphological traits at seedling and maturity stages under heat stress condition in progeny of heat exposure plants from flowering to maturity stages compared to progeny of non-heat prepared plants.

\section{MATERIALS AND MTHODS}

\subsection{The plant material and heat stress priming:}

The initial plant material used in the present study consisted of seven genotypes of bread wheat (Triticum aestivum L.) and three genotypes of durum wheat (Triticum durum L.) in Table 1, were varied in their yield performance under heat stress. The field treatment was carried out at the Experimental Farm of Faculty of Agriculture, Assiut University, Egypt. In 2016/2017 winter season, seeds of the ten genotypes were planted on $21^{\text {st }}$ November as an optimal sowing date (non-primed genotypes, $\mathrm{NH}$ ) and $21^{\text {st }}$ January as a late sowing date (heat primed genotypes, $\mathrm{PH}$ ) to expose genotypes to high temperatures from inception of the flowering stage. At maturity, seeds from both sowing dates were harvested.

Table 1. Pedigree of durum and bread wheat genotypes used in the study.

\begin{tabular}{|c|c|c|c|}
\hline & Name & Code & Pedigree \\
\hline \multirow{3}{*}{ 窇 } & BANI SEWEF 1 & G1 & JO"S"/AA"S"/FG"S" \\
\hline & SVEVO & G2 & Cimmyt's Line / Zenit \\
\hline & WK-12 & G3 & $\begin{array}{c}\text { The landraces were originally collected from farmers' fields near Dandara Temple at Qena } \\
\text { Governorate in } 1993 \text { (Omara, 1994) and were grown since then every year in order to ascertain } \\
\text { the stability of the black glume character. }\end{array}$ \\
\hline \multirow{7}{*}{ 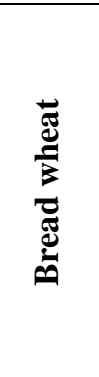 } & GIZA 168 & G4 & MIL/BUC//SERI \\
\hline & L.S.15 (Long spike 15) & G5 & $\begin{array}{c}\text { An advanced long-spike inbred line (F14) derived from a cross among landraces collected from } \\
\text { stress areas in Upper Egypt (Omara,1994) }\end{array}$ \\
\hline & Sonora 64 & G6 & YAKTANA-54//NORIN-10/BREVOR/3/2*YAQUI-54 \\
\hline & Line 6 & G7 & $\begin{array}{l}\text { Advanced long spike, short statured inbred line derived from a cross between two landraces } \\
\text { collected from dry areas in Upper Egypt (Omara, 1994) }\end{array}$ \\
\hline & L.S.16 (Long spike 16) & G8 & $\begin{array}{c}\text { An advanced long-spike inbred line (F14) derived from a cross among landraces collected from } \\
\text { stress areas in Upper Egypt (Omara,1994) }\end{array}$ \\
\hline & $1 * 15$ & G9 & $\begin{array}{l}\text { Advanced breeding line derived from inter population-interenvironmental cross between early } \\
\text { segregates selected in two contrasting environments }\end{array}$ \\
\hline & SIDS 1 & G10 & HD2173/PAVON"S"//1158.57/MAYA 74 "S" \\
\hline
\end{tabular}

\subsection{Estimate of morpho-phosiological traits at seedling stage:}

Seeds harvested from each genotype in optimal and lates sowing date were divided into two groups: The first group was sown in high temperature environment in incubation at a day/night temperature of $35-37^{\circ} \mathrm{C}$, includes the seeds of each genotype yielded under the optimal (non-priming heat stress, $\mathrm{NH}$ ) and late sowing date (priming heat stress, $\mathrm{PH}$ ) conditions. The second group was sown in optimal temperature at $20 \pm 2^{\circ} \mathrm{C}$, includes the seeds of each genotype obtained under the optimal (non-priming heat stress, NC) and late sowing date (priming heat stress, PC) conditions. In both two experiments, the seeds of each genotype generated under optimal and late sowing date; were immersed in sodium hypochlorite for $5 \mathrm{~min}$, then washed three times with distilled water and allowed to germinate in aluminum trays of $30 \mathrm{~cm}$ wide $\times 60 \mathrm{~cm}$ long $\times 7 \mathrm{~cm}$ deep, which were filled with sterilized sand with depth of $5 \mathrm{~cm}$. All experiments were conducted in a randomized complete block design with three replicates in a growth chamber under dark conditions for the first three days. Seedlings were harvested at the $10^{\text {th }}$ day and separated from the remaining seeds. Growth parameters at seedling stage namely; \% of germination $(\% \mathrm{G})$, shoot length, $\mathrm{cm}$ (Sh.L) and root length, cm (R.L), were measured on 10 plants per replicate for each genotype within each group $(\mathrm{NH}, \mathrm{PH}$, $\mathrm{NC}$ and PC) as well as chlorophyll a and b contents (Chl a,b) and percent tetrazolium chloride reduction (\%TTC) were estimated.

\subsection{Tetrazolium chloride (\% TTC) reduction and chlorophyll a, b content $(\mathrm{Chl} \mathbf{a}, \mathrm{b})$ :}

Both \% TTC and Chl a, b measurements were measured on individual plant bases. From each genotype within each group, 10 plants were evaluated for \% TTC reduction using the method of Ibrahim and Quick (2001). The level of acquired high temperature tolerance was determined by measuring the percentage reduction of TTC to formazan using the following 
formula: $\% \mathrm{TTC}=(\mathrm{ODh} / \mathrm{ODc}) \times 100$

where, ODh refers to the mean optical density $(530 \mathrm{~nm})$ values for the heat-stressed set $\left(49{ }^{\circ} \mathrm{C}\right.$ for $\left.90 \mathrm{~min}\right)$, and ODc refers to the mean optical density for the control set $\left(25^{\circ} \mathrm{C}\right.$ for $\left.90 \mathrm{~min}\right)$.

Chlorophyll $\mathrm{a}$ and $\mathrm{b}$ content was estimated following the method of Arnon (1949).

\subsection{Estimate of morpho-physiological traits at} flowering and maturity stage:

In 2017/2018 season, the seeds harvested from each genotype in optimal and late sowing date were divided into two groups: The first group was sown in optimal (20 November, control) and second group was sown in late (20 January, heat stress) sowing date in the Farm of Faculty of Agriculture, Assiut University, Egypt. Both experiments were included on the seeds of each genotype generated under the optimal $(\mathrm{NH})$ and late sowing date $(\mathrm{PH})$ conditions. The experimental design was RCBD in three replications. Each wheat genotype was represented in each block by a row of 10 plants spaced $25 \mathrm{~cm}$ apart within rows set $30 \mathrm{~cm}$ from each other. At flowering stage, relative water content (RWC) and the percent of cell membrane thermostability (\%CMS) were estimated. In addition, grain yield per plant (g) and 1000 grain weight (g) were measured at the maturity stage in the two sowing dates. Relative water content (\%) was calculated using the formula proposed by Ritchier et al. (1990). Cell membrane thermo-stability (\%CMS) assay was performed according to the method described by Fokar et al. (1998).

The recorded maximum air temperatures at the experimental site during March and April of the three consecutive seasons are illustrated graphically in Figure 1 (weather reports in Assiut, https//: W under ground.com) several waves of heat $\left(>34 \mathrm{C}^{\circ}\right)$ characterized the weather of March whereas stronger heat waves $\left(>38 \mathrm{C}^{\circ}\right)$ prevailed during April especially in 2017 and 2018 which coincided with the preflowering stage.

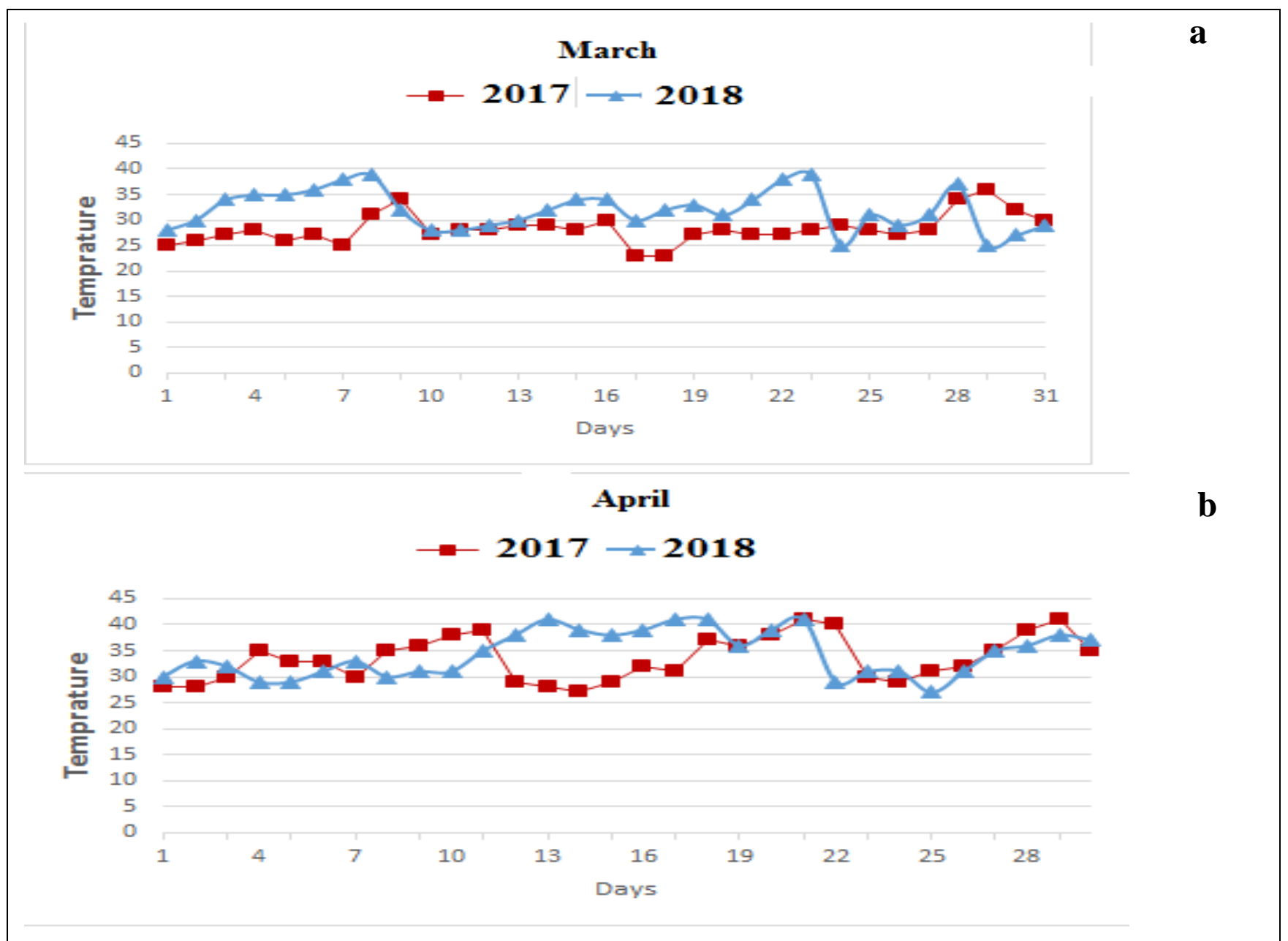

Fig. 1. The recorded maximum air temperature at the experimental site in March (a) and April (b) of the two seasons 2017 and 2018. 


\subsection{Phenotypic data analysis:}

Significantly different means of the results were separated at $\mathrm{P}<0.05$ by Fisher's Least Significant Difference (LSD) and Duncan's multiple range test. Combined analyses of variances across control and heat stress environments and between priming heat $(\mathrm{P})$ and non-priming $(\mathrm{N})$ were used, the broad-sense heritability $\left(h^{2} B\right)$ was estimated using the combined analyses of variances between control and heat stress for each separated group of genotypes primed and non-primed. The broad-sense heritability of a trait was computed using the formula described by Nyquist (1991). T-test was used to significant differences showing between primed plants and non-primed plants for each genotype.

\section{RESULTS}

3.1. Progeny performance of heat primed and nonprimed genotypes at seedling stage:

Progeny means of heat primed and non-primed genotypes under control and heat stress are presented in Table 2. Shoot length ranged from 7.13 (G1) to 17.68 (G9) with an average of $13.12(\mathrm{~cm})$ in progeny of heat primed plants under heat stress $(\mathrm{PH})$ conditions. Furthermore, the shoot length values extended from 10.12 (G6) to 16.40 (G9) with an average of $12.88 \mathrm{~cm}$ in progeny of non-heat primed genotypes under heat stress $(\mathrm{NH})$. As well as, the reduction in shoot length due to heat stress was $19.90 \%$ in the progeny of $\mathrm{PH}$ genotypes compared to $23.79 \%$ in the progeny of $\mathrm{NH}$ genotypes. The chlorophyll a content ranged from 3.87 (G6) to 11.11 (G9) with an average of $6.97 \mu \mathrm{gg}^{-1}$ in the progeny of $\mathrm{PH}$ genotypes, whereas in the progeny of $\mathrm{NH}$ genotypes it ranged from 3.42 (G5) to 9.28 (G4) with an average of $6.23 \mu \mathrm{gg}^{-1}$ while, chlorophyll b content decreased in the progeny of $\mathrm{NH}$ genotypes $(6.58 \%)$ than in the progeny of PH genotypes $(6.13 \%)$ under heat stress condition. The progeny of $\mathrm{PH}$ genotypes showed a \%TTC reduction from $28.21 \%$ (G7) to $78.40 \%$ (G9) with an average of $64.08 \%$. Moreover, the \% TTC reduction in progeny of $\mathrm{NH}$ genotypes extended from $37.28 \%$ (G7) to $66.80 \%$ (G8) with an average of $51.94 \%$. Under heat stress condition, the amount of \% TTC reduction increased amount of (29.69\%) in progeny of $\mathrm{PH}$ genotypes, whereas it increased by $26.59 \%$ in the progeny of $\mathrm{NH}$ genotypes. But the high increase of \% TTC reduction was obtained for progeny of $\mathrm{PH}$ which amounted to $56.18 \%$ compared with the progeny of NC genotypes, indicating that the increase due to effects of environmental epigenetics and inherited epigenetically.

\subsection{Progeny performance of heat primed and non-} primed genotypes at maturity stage:
Progeny means of heat primed and non-primed plants under both favorable and heat stress conditions were shown in Table 3 . The percent of cell membrane thermo-stability (\%CMS) mean values ranged from 21.99 (G10) to $58.28 \%$ (G9) with an average of $41.91 \%$ in progeny of $\mathrm{NH}$ genotypes, while in progeny of $\mathrm{PH}$ genotypes extended from 22.41 (G7) to $81.47 \%$ (G9) with an average of $48.01 \%$.

The \% CMS in progeny of $\mathrm{PH}$ genotypes increased by $14.56 \%$ more than the progeny of NH genotypes. In the progeny of $\mathrm{PH}$ genotypes, grain yield per plant extended from 21.18 (G1) to $48.89 \mathrm{~g}$ (G3) with an average of $33.21 \mathrm{~g}$, whereas the 1000 grain weight ranged from $38.93(\mathrm{G} 4)$ to $50.53 \mathrm{~g}(\mathrm{G} 2)$ with an average overall of $44.72 \mathrm{~g}$. The grain yield per plant and 1000 grain weight decreased by 55.62 and $10.15 \%$, respectively, in progeny of $\mathrm{PH}$ genotypes compared with 62.73 and $11.635 \%$ in the progeny of $\mathrm{NH}$ genotypes. The lower values of heat susceptibility index (HSI) were obtained for all genotypes, in progeny $\mathrm{PH}$ genotypes than those observed in progeny of $\mathrm{NH}$ genotypes except G4 and G10 (Table 3 .)

The combined analysis of variance for all seedling and maturity traits, in progeny of heat primed and nonprimed genotypes were shown in Table 4. High significant differences were obtained between control and heat stress environments for all studied traits at seedling and maturity stages except relative water content at maturity stage. Adding, highly significant differences were observed among genotypes for all studied traits. Highly and significant differences were observed between progeny of primed $(\mathrm{P})$ and nonprimed $(\mathrm{N})$ genotypes for chlorophyll a and $\mathrm{b}$ contents and \% TTC reduction at seedling stage and for \%CMS, grain yield per plant and 1000 grain weight at maturity stage. Moreover, the shoot length, chlorophyll a content, relative water content, \%CMS and 1000 grain weight showed significant differences between durum (D) and bread wheat (B) genotypes in progeny of primed and non-primed genotypes.

The variances estimated were higher in progeny of $\mathrm{PH}$ genotypes than observed in progeny of $\mathrm{NH}$ genotypes for all studied traits at seedling and maturity stages (Table 5). The $\mathrm{PH} / \mathrm{NH}$ mean ratio was higher than one for all studied traits except the $\%$ germination, root length and relative water content .

The analysis of variance of four treatment combinations (NC, PC, $\mathrm{NH}$ and $\mathrm{PH}$ ) for all studied traits at seedling and maturity stages are displayed in 
Mahmoud A. El-Rawy., 2020

Table 2. Progeny means of heat primed and non-primed plants for seedling traits under high and optimal temperature conditions.

\begin{tabular}{|c|c|c|c|c|c|c|c|c|c|c|c|c|c|c|}
\hline & \multirow{2}{*}{ 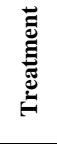 } & \multirow{2}{*}{ 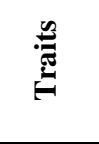 } & \multicolumn{12}{|c|}{ Genotypes } \\
\hline & & & G1 & G2 & G3 & G4 & G5 & G6 & G7 & G8 & G9 & G10 & Mean & $\operatorname{LSD}_{(0.05)}$ \\
\hline \multirow{12}{*}{ 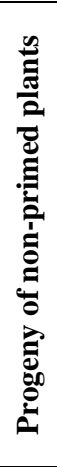 } & \multirow{6}{*}{ 宊 } & $\% \mathrm{G}$. & 100.0 & 100.0 & 92.86 & 100.0 & 85.71 & 78.57 & 100.0 & 100.0 & 85.71 & 57.14 & 90.00 & 16.73 \\
\hline & & Sh. L & 16.50 & 18.35 & 15.52 & 16.12 & 18.34 & 14.79 & 16.83 & 18.00 & 17.00 & 17.54 & 16.90 & 2.16 \\
\hline & & R. L & 12.07 & 12.51 & 7.45 & 9.13 & 7.90 & 9.33 & 7.71 & 8.79 & 8.75 & 9.88 & 9.35 & 3.52 \\
\hline & & Chl. a & 19.59 & 18.77 & 29.44 & 20.45 & 17.74 & 21.58 & 22.40 & 16.37 & 19.63 & 22.14 & 20.81 & 3.70 \\
\hline & & Chl. b & 11.84 & 10.48 & 14.37 & 12.52 & 10.51 & 13.36 & 13.04 & 9.08 & 10.56 & 11.46 & 11.72 & 2.14 \\
\hline & & \% TTC & 35.03 & 42.02 & 50.35 & 25.01 & 32.55 & 58.40 & 51.26 & 44.98 & 26.35 & 44.38 & 41.03 & 20.60 \\
\hline & \multirow{6}{*}{ 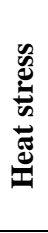 } & $\%$ G. & 85.71 & 78.57 & 42.86 & 78.57 & 71.43 & 88.90 & 71.43 & 92.86 & 85.71 & 78.57 & 77.46 & 22.39 \\
\hline & & Sh. L & 13.58 & 14.57 & 14.50 & 12.07 & 10.20 & 10.12 & 14.60 & 10.14 & 16.40 & 12.63 & 12.88 & 3.75 \\
\hline & & R. $L$ & 4.17 & 5.28 & 3.58 & 3.72 & 3.15 & 3.53 & 3.00 & 3.14 & 6.22 & 3.65 & 3.94 & 1.65 \\
\hline & & Chl. a & 4.88 & 5.04 & 8.05 & 9.28 & 3.42 & 3.58 & 8.01 & 5.34 & 7.43 & 7.28 & 6.23 & 1.15 \\
\hline & & Chl. b & 3.15 & 2.93 & 5.41 & 4.78 & 2.54 & 2.56 & 5.04 & 3.48 & 5.30 & 4.87 & 4.01 & 1.92 \\
\hline & & \% TTC & 60.04 & 60.98 & 48.89 & 46.07 & 62.76 & 44.51 & 37.28 & 66.80 & 30.37 & 61.65 & 51.94 & 16.19 \\
\hline \multirow{12}{*}{ 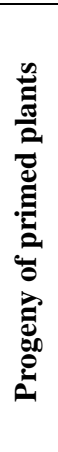 } & \multirow{6}{*}{ 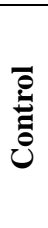 } & $\%$ G. & 85.71 & 85.71 & 71.43 & 42.86 & 71.43 & 71.43 & 57.14 & 71.43 & 85.71 & 78.57 & 72.14 & 15.64 \\
\hline & & Sh. L & 12.63 & 18.37 & 18.23 & 14.75 & 16.63 & 16.38 & 15.63 & 18.72 & 18.13 & 14.33 & 16.38 & 3.60 \\
\hline & & R. L & 5.38 & 9.73 & 7.98 & 6.22 & 7.38 & 11.38 & 6.75 & 8.23 & 7.63 & 7.50 & 7.82 & 2.16 \\
\hline & & Chl. a & 18.92 & 20.21 & 29.39 & 19.93 & 17.81 & 25.34 & 22.57 & 16.17 & 24.29 & 23.59 & 21.82 & 2.98 \\
\hline & & Chl. b & 10.27 & 11.40 & 14.27 & 11.17 & 9.83 & 14.45 & 13.51 & 8.79 & 12.43 & 11.68 & 11.78 & 2.03 \\
\hline & & \% TTC & 52.90 & 61.60 & 53.78 & 49.54 & 30.05 & 53.22 & 51.27 & 42.64 & 55.16 & 43.98 & 49.41 & 14.00 \\
\hline & \multirow{6}{*}{ 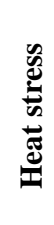 } & $\%$ G. & 28.57 & 85.71 & 50.00 & 85.76 & 48.05 & 86.95 & 43.90 & 71.43 & 78.10 & 24.76 & 60.32 & 30.80 \\
\hline & & Sh. L & 7.13 & 17.65 & 8.17 & 13.92 & 11.33 & 13.74 & 14.41 & 16.40 & 17.68 & 10.75 & 13.12 & 3.87 \\
\hline & & R. $\mathbf{L}$ & 2.75 & 5.48 & 3.38 & 3.75 & 3.75 & 3.95 & 2.83 & 4.40 & 5.79 & 2.25 & 3.83 & 1.71 \\
\hline & & Chl. a & 5.37 & 6.27 & 8.56 & 7.36 & 4.23 & 3.87 & 9.29 & 6.43 & 11.11 & 7.19 & 6.97 & 2.22 \\
\hline & & Chl. b & 3.65 & 3.37 & 5.66 & 4.58 & 3.19 & 2.96 & 6.04 & 4.21 & 6.89 & 5.02 & 4.56 & 1.39 \\
\hline & & \% TTC & 73.42 & 75.71 & 37.82 & 56.66 & 74.37 & 73.93 & 28.21 & 74.82 & 78.40 & 67.42 & 64.08 & 14.39 \\
\hline
\end{tabular}

Table 3. Progeny means of heat primed and non-primed plants for maturity traits under control and heat stress conditions as well as heat susceptibility index.

\begin{tabular}{|c|c|c|c|c|c|c|c|c|c|c|c|c|c|c|}
\hline & \multirow{2}{*}{ 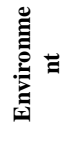 } & \multirow{2}{*}{ 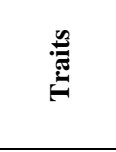 } & \multicolumn{12}{|c|}{ Genotypes } \\
\hline & & & G1 & G2 & G3 & G4 & G5 & G6 & G7 & G8 & G9 & G10 & Mean & $\operatorname{LSD}_{(0.05)}$ \\
\hline \multirow{9}{*}{ 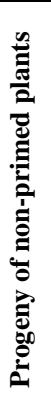 } & \multirow{4}{*}{ 它 } & RWC & 93.11 & 90.82 & 80.87 & 81.52 & 86.32 & 89.19 & 85.74 & 91.34 & 75.60 & 80.21 & 85.47 & 2.87 \\
\hline & & $\%$ CMS & 28.84 & 25.19 & 26.47 & 26.03 & 23.50 & 33.57 & 32.45 & 25.93 & 30.38 & 25.91 & 27.83 & 1.66 \\
\hline & & $1000 \mathrm{KW}$ & 54.77 & 52.03 & 54.17 & 33.50 & 46.93 & 42.77 & 49.00 & 49.93 & 51.23 & 54.17 & 48.85 & 3.26 \\
\hline & & G. yield & 86.59 & 83.18 & 82.85 & 41.93 & 105.8 & 60.06 & 119.8 & 101.8 & 55.84 & 76.46 & 81.44 & 12.0 \\
\hline & \multirow{5}{*}{ 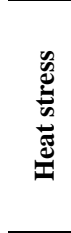 } & RWC & 93.01 & 90.51 & 90.06 & 95.06 & 85.91 & 91.25 & 83.47 & 80.96 & 90.95 & 93.36 & 89.45 & 2.27 \\
\hline & & \% CMS & 55.28 & 34.52 & 38.76 & 47.50 & 45.23 & 43.38 & 28.92 & 45.25 & 58.28 & 21.99 & 41.91 & 5.59 \\
\hline & & $1000 \mathrm{KW}$ & 42.37 & 46.93 & 45.47 & 36.33 & 38.53 & 40.03 & 42.40 & 41.73 & 45.50 & 52.37 & 43.17 & 2.30 \\
\hline & & G. yield & 16.98 & 27.25 & 39.03 & 25.60 & 28.76 & 29.11 & 36.17 & 31.05 & 29.62 & 39.98 & 30.35 & 3.40 \\
\hline & & HSI & 1.32 & 1.10 & 0.87 & 0.64 & 1.19 & 0.84 & 1.14 & 1.14 & 0.77 & 0.78 & & \\
\hline \multirow{9}{*}{ 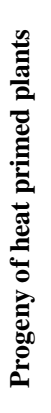 } & \multirow{4}{*}{ نَّ } & RWC & 89.26 & 93.63 & 84.94 & 81.98 & 87.84 & 84.75 & 87.98 & 93.22 & 86.62 & 84.27 & 87.45 & 1.89 \\
\hline & & $\%$ CMS & 28.21 & 27.39 & 24.68 & 25.02 & 26.02 & 27.87 & 29.31 & 27.80 & 49.37 & 24.86 & 29.05 & 3.65 \\
\hline & & $1000 \mathrm{KW}$ & 54.67 & 48.13 & 62.47 & 33.37 & 47.20 & 43.27 & 52.03 & 49.00 & 49.87 & 57.70 & 49.77 & 3.98 \\
\hline & & G. yield & 80.90 & 72.31 & 79.92 & 39.22 & 104.1 & 56.10 & 99.94 & 90.08 & 47.01 & 78.73 & 74.83 & 10.8 \\
\hline & \multirow{5}{*}{ 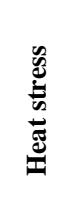 } & RWC & 96.74 & 88.64 & 88.17 & 83.24 & 79.52 & 91.24 & 84.10 & 71.47 & 84.16 & 92.08 & 85.94 & 3.56 \\
\hline & & \% CMS & 66.21 & 47.00 & 46.40 & 43.25 & 42.78 & 55.29 & 22.41 & 43.29 & 81.47 & 32.01 & 48.01 & 8.30 \\
\hline & & $1000 \mathrm{KW}$ & 42.60 & 50.83 & 39.23 & 38.93 & 39.43 & 40.53 & 50.93 & 45.70 & 48.47 & 50.57 & 44.72 & 2.57 \\
\hline & & G. yield & 21.18 & 37.84 & 48.86 & 21.65 & 40.25 & 31.94 & 35.33 & 38.64 & 32.89 & 23.52 & 33.21 & 4.47 \\
\hline & & HSI & 1.27 & 0.82 & 0.67 & 0.77 & 1.06 & 0.74 & 1.11 & 0.98 & 0.52 & 1.21 & & \\
\hline
\end{tabular}


Scientific Journal of Agricultural Sciences 2 (2): 161-173, 2020

Table 4. Means of seedling and maturity traits for NC, NH, PC and PH treatments combinations as well as variance, \% CV and PH/NH Ratio.

\begin{tabular}{|c|c|c|c|c|c|c|c|c|c|}
\hline \multirow[b]{2}{*}{ Traits } & \multicolumn{4}{|c|}{ Mean } & \multicolumn{2}{|c|}{ Variance } & \multicolumn{2}{|c|}{$\% \mathrm{CV}$} & \multirow{2}{*}{$\begin{array}{c}\text { PH/NH } \\
\text { Ratio }\end{array}$} \\
\hline & $\mathbf{N C}$ & NH & PC & PH & NH & PH & NH & PH & \\
\hline \multicolumn{10}{|c|}{ Seedling traits } \\
\hline$\% \mathbf{G}$. & $90.00^{\mathrm{a}}$ & $77.46^{\mathrm{b}}$ & $72.14^{\mathrm{c}}$ & $60.32^{d}$ & 197.53 & 582.20 & 18.14 & 40.00 & 0.78 \\
\hline Sh. L & $16.90^{\mathrm{a}}$ & $12.88^{\mathrm{b}}$ & $16.38^{\mathrm{a}}$ & $13.12^{\mathrm{b}}$ & 4.93 & 13.77 & 17.23 & 28.29 & 1.02 \\
\hline R. L & $9.35^{\mathrm{a}}$ & $3.94^{\mathrm{c}}$ & $7.82^{\mathrm{b}}$ & $3.83^{\mathrm{c}}$ & 1.07 & 1.31 & 26.22 & 29.85 & 0.97 \\
\hline Chl. a & $20.81^{\mathrm{b}}$ & $6.23^{\mathrm{d}}$ & $21.82^{\mathrm{a}}$ & $6.97^{\mathrm{c}}$ & 4.14 & 5.08 & 32.66 & 32.34 & 1.12 \\
\hline Chl. b & $11.72^{\mathrm{a}}$ & $4.01^{\mathrm{c}}$ & $11.78^{\mathrm{a}}$ & $4.56^{\mathrm{b}}$ & 1.39 & 1.76 & 29.38 & 29.12 & 1.14 \\
\hline$\% \mathrm{TTC}$ & $41.03^{\mathrm{d}}$ & $51.94^{\mathrm{b}}$ & $49.41^{\mathrm{c}}$ & $64.08^{a}$ & 150.96 & 309.81 & 23.66 & 27.47 & 1.23 \\
\hline \multicolumn{10}{|c|}{ Maturity traits } \\
\hline RWC & $85.47^{\mathrm{c}}$ & $89.45^{\mathrm{a}}$ & $87.45^{\mathrm{b}}$ & $85.94^{c}$ & 20.76 & 51.00 & 5.09 & 8.31 & 0.96 \\
\hline$\%$ CMS & $27.83^{\mathrm{c}}$ & $41.91^{\mathrm{b}}$ & $29.05^{\mathrm{c}}$ & $48.01^{\mathrm{a}}$ & 125.82 & 276.79 & 26.76 & 34.65 & 1.15 \\
\hline $1000 \mathrm{KW}$ & $48.85^{\mathrm{b}}$ & $43.17^{\mathrm{d}}$ & $49.77^{\mathrm{a}}$ & $44.72^{\mathrm{c}}$ & 21.20 & 26.52 & 10.67 & 11.52 & 1.04 \\
\hline G. Yield & $81.44^{\mathrm{a}}$ & $30.35^{\mathrm{d}}$ & $74.83^{\mathrm{b}}$ & $33.21^{\mathrm{c}}$ & 46.42 & 80.42 & 22.45 & 27.00 & 1.09 \\
\hline
\end{tabular}

NC, progeny of non-primed plants under control.

PC, progeny of heat primed plants under control.

$\mathrm{NH}$, progeny of non-primed plants under heat stress.

$\mathrm{PH}$, progeny of heat primed plants under heat stress

Values with the same letter are not significantly different according to Duncan's multiple range test at $\mathrm{P}=0.05$

Table 5. Mean squares of analysis of variance among NC, NH, PC and PH treatments combinations for seedling and maturity traits.

\begin{tabular}{|c|c|c|c|c|c|c|c|c|c|c|c|}
\hline \multicolumn{8}{|c|}{ Seedling traits } & \multicolumn{4}{|c|}{ Flowering and maturity traits } \\
\hline S. O. V. & d. $\mathbf{f}$ & $\% \mathbf{G}$ & Sh. L & R. L & Chl. a & Chl. b & $\%$ TTC & RWC & $\begin{array}{c}\% \\
\text { CMS }\end{array}$ & $\begin{array}{l}1000 \\
\text { KW }\end{array}$ & G. Yield \\
\hline Rep. & 2 & 17.82 & 0.21 & $0.024 *$ & 0.07 & 0.004 & 0.23 & 0.25 & 1.47 & 0.02 & 0.57 \\
\hline Treatments & 3 & $454.61 * *$ & $\begin{array}{c}13.44 \\
* *\end{array}$ & $\begin{array}{c}22.71 \\
* *\end{array}$ & $217.42 * *$ & $\begin{array}{c}55.94 \\
* *\end{array}$ & $240.72 * *$ & $\begin{array}{c}9.67 \\
* *\end{array}$ & $\begin{array}{c}211.7 \\
* *\end{array}$ & $\begin{array}{c}30.42 \\
* *\end{array}$ & $2174.4 * *$ \\
\hline Error & 6 & 9.88 & 0.23 & 0.003 & 0.05 & 0.13 & 2.92 & 0.30 & 2.30 & 0.15 & 3.29 \\
\hline
\end{tabular}

*, ** Significant differences at $\mathrm{P}<0.05$ and $\mathrm{P}<0.01$, respectively

Table 6. Mean squares of analysis of variance for seedling and maturity traits among progeny of non-primed (N) and primed plants (P) in durum (D) and bread (B) wheat under control and heat stress conditions.

\begin{tabular}{|c|c|c|c|c|c|c|c|}
\hline \multicolumn{8}{|c|}{ Seedling traits } \\
\hline S. O.V. & D.F. & $\% \mathbf{G}$. & Sh. L & R. $\mathbf{L}$ & Chl. a & Chl. b & $\%$ TTC \\
\hline Tr. & 1 & $4449.53 * *$ & $398.37 * *$ & $661.71 * *$ & $6499.23 * *$ & $1673.59 * *$ & $4525.73 * *$ \\
\hline R/Tr. & 4 & $156.88^{*}$ & 1.21 & 0.02 & 0.69 & $1.48^{*}$ & 17.09 \\
\hline Geno. & 19 & $1204.25 * *$ & $23.47 * *$ & $9.33 * *$ & $38.22 * *$ & $9.00 * *$ & $657.97 * *$ \\
\hline N vs $P$ & 1 & $9185.00 * *$ & 0.65 & $20.36 * *$ & $22.96 * *$ & $2.81 *$ & $2663.42 * *$ \\
\hline Among N & 9 & $563.11 * *$ & $9.40 * *$ & $8.47 * *$ & $35.99 * *$ & $7.99 * *$ & $607.54 * *$ \\
\hline D vs B & 1 & 4.07 & $9.63 * *$ & $19.14 * *$ & $15.43 * *$ & 0.72 & $676.40 * *$ \\
\hline Among D & 2 & $1096.94 * *$ & 4.10 & $18.81 * *$ & $89.37 * *$ & $16.54 * *$ & 32.19 \\
\hline Among B & 6 & $478.35^{* *}$ & $11.13 * *$ & $3.25 * *$ & $21.63 * *$ & $6.36 * *$ & $787.85^{* *}$ \\
\hline Among $P$ & 9 & $958.63 * *$ & $40.08 * *$ & $8.95 * *$ & $42.14 * *$ & $10.70 * *$ & $485.57 * *$ \\
\hline D vs B & 1 & 67.80 & $28.26^{* *}$ & 0.05 & $3.97 * *$ & 0.11 & 20.76 \\
\hline Among D & 2 & $1454.08 * *$ & $100.33^{* *}$ & $18.86^{* *}$ & $80.75 * *$ & $15.85 * *$ & $784.82 * *$ \\
\hline Among $B$ & 6 & $941.97 * *$ & $21.96 * *$ & $4.76^{* *} *$ & $23.75 * *$ & $7.16^{* *}$ & $308.86^{* *}$ \\
\hline $\mathbf{T} \times \mathbf{G}$ & 19 & $929.62 * *$ & $11.18^{* *}$ & $7.14 * *$ & $25.23 * *$ & $6.77 * *$ & $828.44 * *$ \\
\hline Error & 76 & 57.32 & 1.44 & 0.63 & 0.82 & 0.42 & 30.69 \\
\hline \multicolumn{8}{|c|}{ Flowering and maturity traits } \\
\hline S. O.V. & D.F. & RWC & & $\%$ CMS & $1000 \mathrm{KW}$ & & G. Yield \\
\hline En. & 1 & 45.70 & & $6286.12 * *$ & $863.50 * *$ & & $64452.92 * *$ \\
\hline R/En. & 4 & 1.53 & & 15.34 & 0.41 & & 16.21 \\
\hline Geno. & 19 & $62.12 * *$ & & $562.82 * *$ & $161.15 * *$ & & $1006.66 * *$ \\
\hline N vs $\mathbf{P}$ & 1 & 17.81 & & $63.73^{*}$ & $46.00^{* *}$ & & $105.68 *$ \\
\hline Among $\mathbf{N}$ & 9 & $55.71 * *$ & & $329.81 * *$ & $164.32 * *$ & & $1045.71 * *$ \\
\hline D vs B & $\mathbf{1}$ & $132.16^{* *}$ & & $583.76 * *$ & $276.74 * *$ & & 0.17 \\
\hline Among D & 2 & $90.45^{* *}$ & & 31.35 & 2.51 & & $128.30 * *$ \\
\hline Among B & 6 & 31.40 & & $386.97 * *$ & $199.52 * *$ & & $1525.76^{* *}$ \\
\hline Among $\mathbf{P}$ & 9 & $73.45 * *$ & & $851.29 * *$ & $170.77 * *$ & & $1067.73 * *$ \\
\hline D vs B & 1 & $321.80 * *$ & & $328.61 * *$ & $149.21 * *$ & & $203.79 * *$ \\
\hline Among D & 2 & $65.96 *$ & & $239.30 * *$ & 7.50 & & $281.27 * *$ \\
\hline Among B & 6 & 23.04 & & $761.60 * *$ & $152.52 * *$ & & $982.58 * *$ \\
\hline $\mathbf{E} \times \mathbf{G}$ & 19 & $120.21 * *$ & & $354.50 * *$ & $60.72 * *$ & & $700.28 * *$ \\
\hline Error & 76 & 17.05 & & 15.40 & 3.03 & & 22.29 \\
\hline
\end{tabular}

*, ** Significant differences at $\mathrm{P}<0.05$ and $\mathrm{P}<0.01$, respectively 
Table 6. Highly significant differences among the treatment combinations were obtained for all traits.

\subsection{Evaluation of stress memory for tested genotypes:}

Among ten genotypes that were used in this study, the genotype G9 displayed high significant differences between progeny of $\mathrm{PH}$ plants and progeny of NH plants in chlorophyll $a$ and $b$ content, \% TTC reduction and \%CMS. Similarly, significant differences between progeny of $\mathrm{PH}$ plants and progeny of $\mathrm{NH}$ plants in G1 and G2 were observed for chlorophyll a and $\mathrm{b}$ content, \% TTC reduction and \%CMS. In addition, the G6 and G10 genotypes revealed significant differences between the progeny of $\mathrm{PH}$ plants and the progeny of NH plants in \% TTC reduction and \%CMS (Figure 2).

Estimated values of broad sense heritability were higher in progeny of $\mathrm{PH}$ genotypes than in progeny of $\mathrm{NH}$ genotypes for all studied traits at seedling and maturity stages, except \% CMS and 1000 grain weight (Figure 3). The high broad sense heritability values were obtained in progeny of $\mathrm{PH}$ genotypes which amounted to 0.63 for $\%$ germination, 0.72 for shoot length, 0.55 for root length, 0.74 for chlorophyll a content, 0.81 chlorophyll $\mathrm{b}$ content and 0.64 for $\%$ TTC reduction at seedling stage. While, at maturity stage, the broad sense heritability values amounted to 0.75 for relative water content, 0.74 for $\%$ CMS, 0.64 for 1000 grain wheat and 0.64 for grain yield per plant. Progeny of $\mathrm{NH}$ genotypes given high to moderate broad sense heritability values i.e. $0.51,0.48$, $0.51,0.63,0.50,0.73,0.23,0.81,0.80,0.43$ for $\%$ germination, shoot length, root length, chlorophyll a content, chlorophyll b content, \% TTC reduction, relative water content, \% CMS, 1000 grain weight and grain yield per plant, respectively.

Denderogram constructed based on genetic distance matrix obtained from morpho-physiological traits for progeny of heat primed $(\mathrm{P})$ and non-primed $(\mathrm{N})$ genotypes under heat stress condition are shown in Figure 4. The denderogram grouped all genotypes into four clusters. The G9 genotype of heat primed plants (G9-P) was separated in a single branch within the dendrogram and it showed the longest genetic distance $(\mathrm{Gd}=4.44)$ when compared with the G9-N (Nonprimed plants). Both G10-P and G1-P genotypes were found in the next cluster and come after G9 in the genetic distance as compared with $\mathrm{G} 10-\mathrm{N}(\mathrm{Gd}=4.15)$ and $\mathrm{G} 1-\mathrm{N}(\mathrm{Gd}=4.00)$. These results were substantiated by the fact that the performance of heat primed progeny of these genotypes was significantly higher than the performance of non-primed progeny in most studied traits under heat stress. These results indicated that heat stress memory was more obvious in these genotypes. Although the G5-P and G5-N genotype showed the shortest genetic distance $(\mathrm{Gd}=2.49)$, the performance of G5-P progeny was higher than G5-N progeny under heat stress as described above, indicating the existence of low level of heat stress memory in G5. The other genotypes were grouped in the last clusters and showed moderate genetic distance reflecting moderate levels of heat stress memory.

\section{DISCUSSION}

High temperature stress is a major constraint to the wheat productivity at all growth stages in most of the cereal growing areas especially the warmer regions of the world like the Mediterranean climate in Egypt (Fischer 1986). The epigenetic mechanisms are involved in the inheritance of tolerance to abiotic stress in trans-generational priming (Balmer et al. 2015). Wherefore in the present study, the effects of stress memory resulting from exposure to the high temperatures were evaluated in progeny of heat-primed genotypes. Under high-temperature stress during seedling stage, the progeny of the heat primed genotypes (PH) showed significantly higher chlorophyll $\mathrm{a}$ and $\mathrm{b}$ content and \% TTC than that of the non-heat primed genotypes ( $\mathrm{NH})$. At maturity stage, under heat stress conditions, the grain yield per plant which is associated with the higher grain weight for progeny of $\mathrm{PH}$ genotypes were higher than that of $\mathrm{NH}$ genotypes. The greater grain weight could be resulted from the higher cell membrane thermo-stability (\%CMS) and \% TTC in the PH genotypes than in the $\mathrm{NH}$ genotypes. These results indicate to, the heat primed effect during the parents inherited for progeny which could enhance their thermo-tolerance.

Heat stress causes damage to cellular function and affects various metabolic bath-ways, especially those relating to photosynthesis, membrane thermostability, starch synthesis (Larkindale and Knight 2002), increase of unsaturated fatty acids and denaturation of proteins caused by high temperature disrupt ion water and organic solute movement among membranes, leading to increased cell membrane damage and in turn, cellular function inhibition (Cossani and Reynolds 2012). Cell membrane thermostability, leaf greenness, Leaf chlorophyll content and canopy temperature have been suggested as elect traits to crops improve of adaptation and yield potential of wheat under high temperature (Pradhan et al. 2020). 
Mahmoud A. El-Rawy., 2020
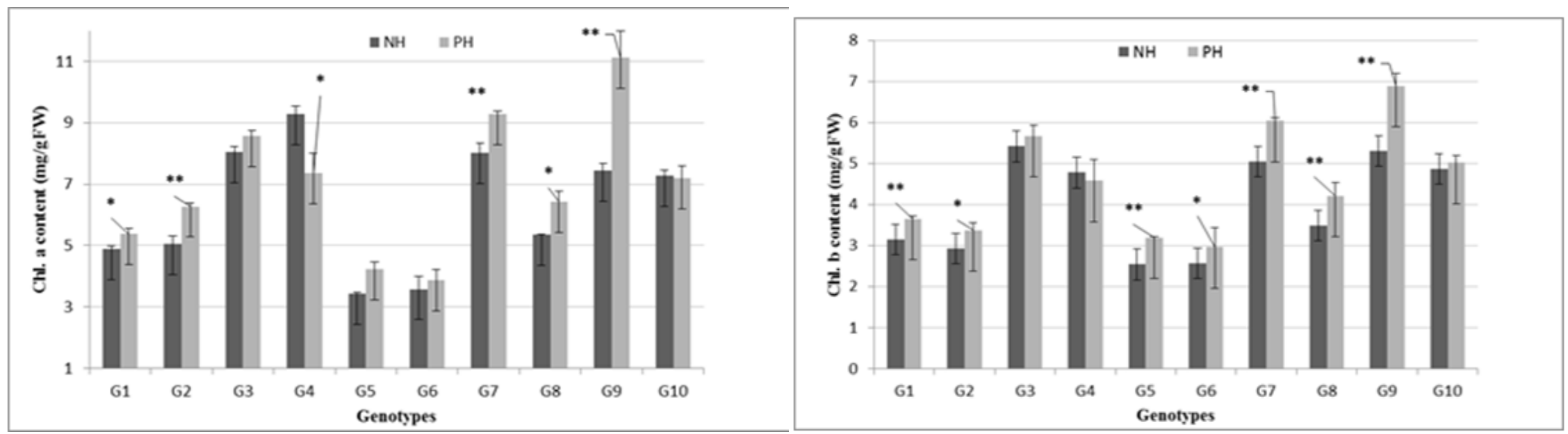

$\mathbf{a}$

b
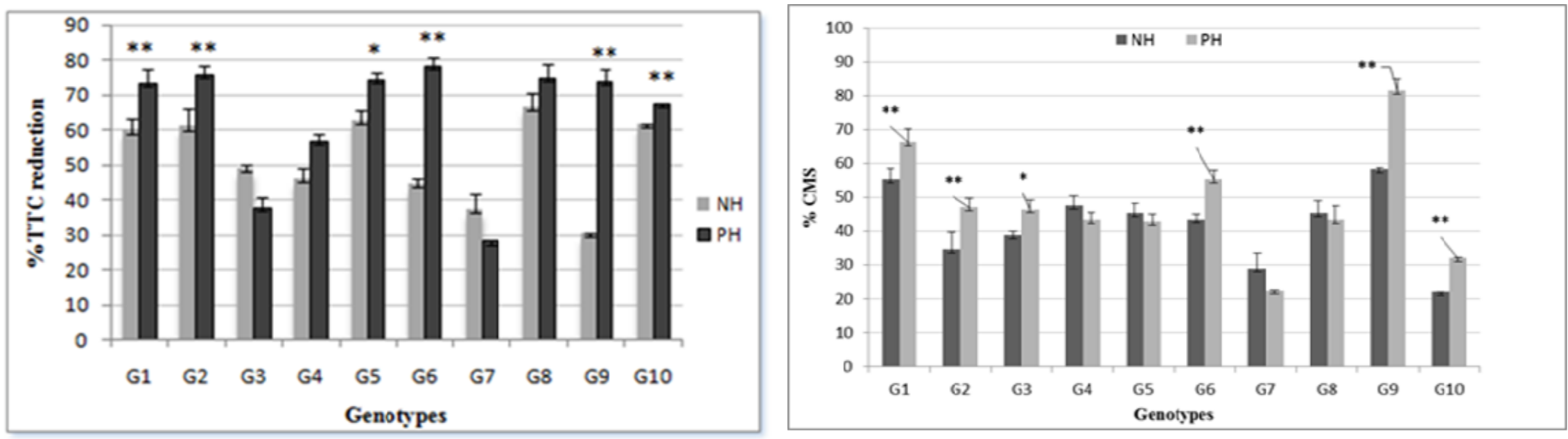

c

d

Fig. 2: Means, error bar and T-test for chlorophyll a (a) and b (b) content, \% TTC reduction (c) and \% CMS (d) in heat-primed (PH) and nonprimed (NH) genotypes. 
Mahmoud A. El-Rawy., 2020

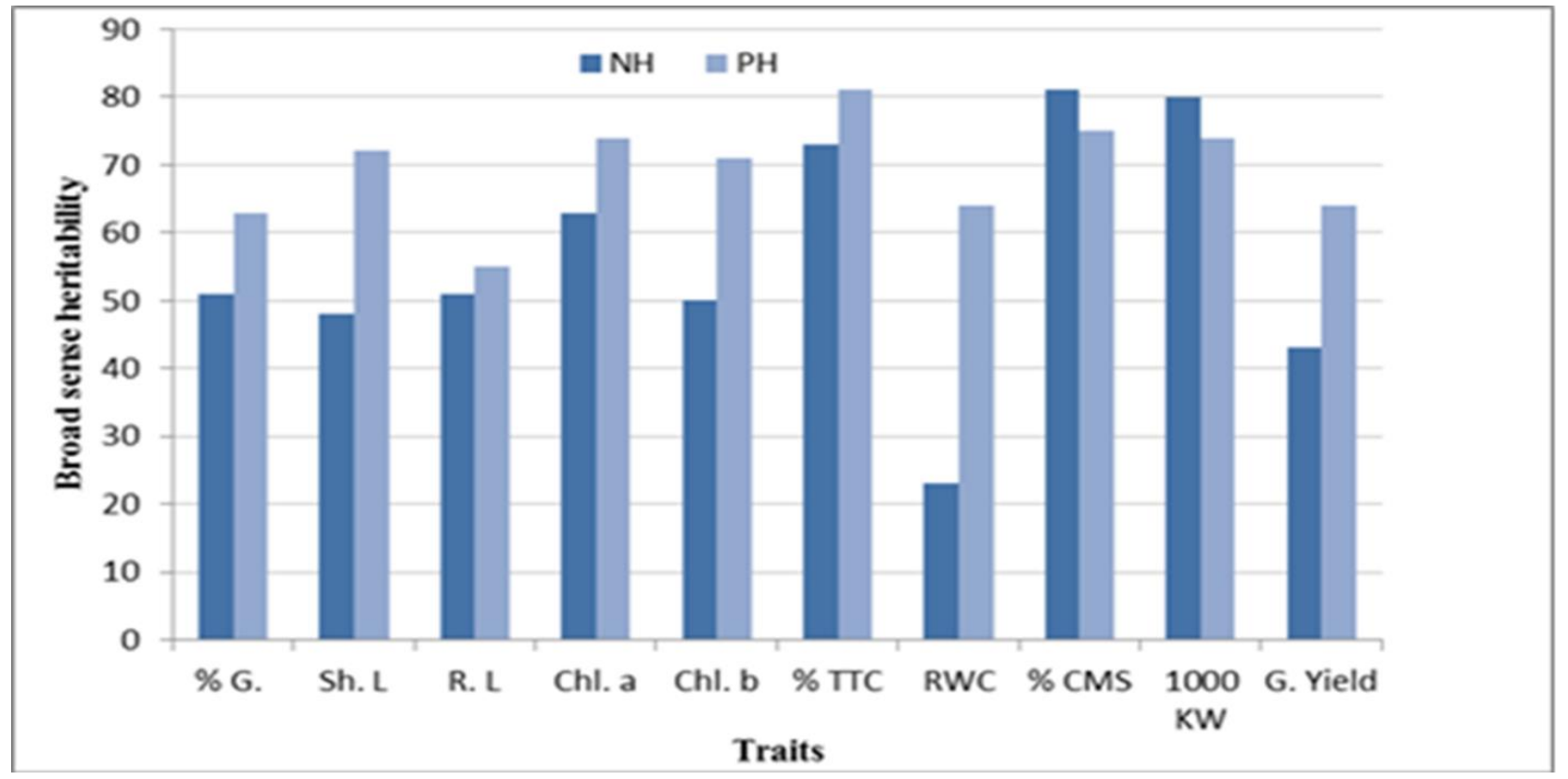

Fig. 3: The broad sense heritability for seedling and maturity traits in heat-primed ( $\mathrm{PH})$ and non-primed $(\mathrm{NH})$ genotypes.

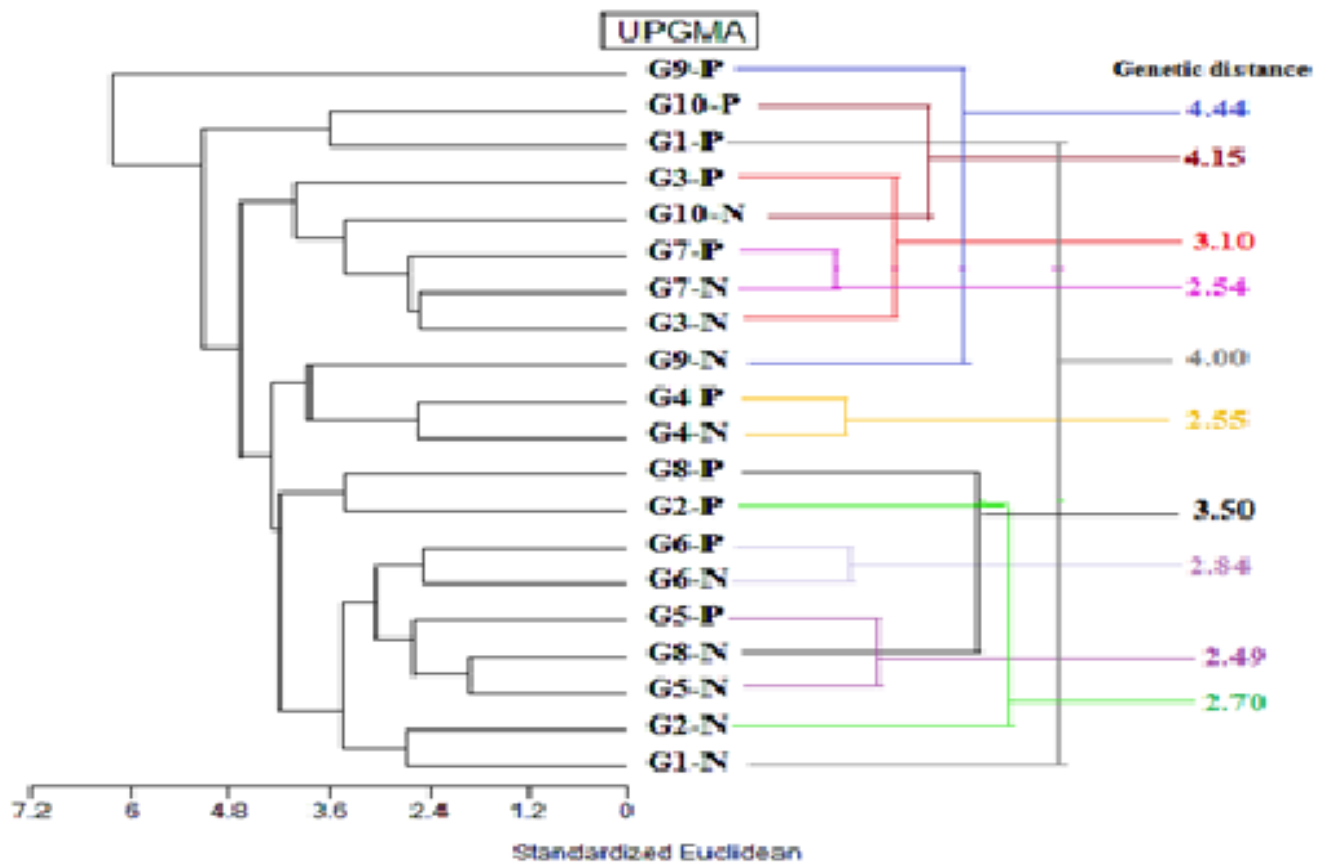

Fig. 4. Dendrogram of genetic distance using morpho-physiological data and genetic distance values between heat-primed plants and non-primed plants for each genotype.

Boyko et al. (2010) found that the progeny of heat primed Arabidopsis plants are more tolerant to many biotic stresses and that the tolerance is associated with substantially modified gene expressions, increased methylation of DNA and increased frequency of homologous recombination. Epigenetics modified like putative nucleic acid methyl transferases and binding proteins, Lysine-specific histone demethylase 1 (LSD1), RNA methyltransferase, ribosomal RNA FtsJlike methyltransferase were expressed much higher levels in the progeny of the heat primed plants than in those of the non-heat primed plants under heat stress at 


\section{Scientific Journal of Agricultural Sciences 2 (2): 161-173, 2020}

post-anthesis in wheat (Wang et al. 2016). The mechanisms of cell membrane thermo-tolerance to heat stress in plants have been detailed, including the heat shock proteins (HSPs), heat shock factors (HSFs), reactive oxygen species (ROS) (Driedonks et al. 2015). HSPs and HSFs play central roles in the thermotolerance of plants. HSFs are the central regulators accountable for the expression of HSP genes. $21 \mathrm{HSFs}$ members are found in The Arabidopsis genome (Baniwal et al. 2004).

High temperature adversely affects photosynthesis in a number of ways (Shah and Paulsen 2003). Photosystem II and Thylakoid membranes are considered the most heat convertible cell components (Ristic et al. 2007). Thylakoid membranes under heat stress showed increased leakiness, swelling, disruption of photosystem II mediated electron transfer, and physical separation of the chlorophyll light harvesting complex II from the photosystem II core complex (Ristic et al. 2008). Thylakoids contain chlorophyll and damage to thylakoids caused by heat leads to chlorophyll loss (Jenner 1994).

High temperature affects morphological traits at every developmental stages in wheat, but the anthesis and pre-flowering stages are more sensitive to heat stress compared to post-flowering stages (Cossani and Reynolds 2012; Prasad et al. 2018). Specifically, short periods under heat stress at the flowering and preflowering stages can reduce yield and grain number per spike. This can be imputed to lower of pollen ability to germinate, and to the rate of growth of pollen tube (Prasad et al. 2018). Recently, emulations based on predictions of future environmental indicated that by year 2100 expected increases in high temperature would lead to mean grain yield reductions of $17.5 \%$ and $7.1 \%$ for spring and winter wheat, respectively (Yang et al. 2017).

In the present study, the variance estimated were higher in the progeny of $\mathrm{PH}$ genotypes than observed in the progeny of NH genotypes for all studied traits at seedling and maturity stages, as well as, the broad sense heritability values estimated were higher in progeny of $\mathrm{PH}$ genotypes than in progeny of $\mathrm{NH}$ genotypes for all studied traits at seedling and maturity stages except \% CMS and 1000 grain weight (Figure 3), indicating the effects of heat stress memory or epigenetic variations inherited in the progeny of $\mathrm{PH}$ genotypes. Latzel et al. (2013) demonstrated that epigenetic variation among inbred lines (RILs) creates diversity in phenotypes, thereby increasing the productivity and stability of plant populations. Epigenetic variation may provide additional sources of diversity within a genotypes that could be captured or created for crop improvement. It will be remarkable to understand the sources of epigenetic variation and the stability of newly formed epigenetic variants transgenerations to fully use the potential of epigenetic variation to improve crops (Springer and Schmitz 2017). Verhoeven et al. (2016) found that the epigenetic modifications has been well established, but still unknown, or to what degree, environmentally induced epigenetic variations are transferred transgenerations. If epigenetic signals making phenotypic diversity are inherited, they can form the basis of adaptive evolutionary change (Kronholm, and Collins 2016). Cubas et al. (1999) identified natural variation attributed to epialleles that affected plant traits such as floral morphology and anthocyanin content which according to Chandler (2007). Vogt (2018) explained the relationship between genetic and epigenetic variation, inheritance of the transgenerational epigenetic and the contribution of epigenetic phenotype variation and environmental adaptation in Marbled crayfish. High broad sense heritability was found for 1000 seeds weight in Helianthus annuus L. by Mostafa (2020).

In this study, G9, G1 and G2 genotypes given significant differences between progeny of $\mathrm{PH}$ plants and progeny of $\mathrm{NH}$ plants in chlorophyll $\mathrm{a}$ and $\mathrm{b}$ content, \% TTC reduction, \%CMS and grain yield per plant. Similarly, highly significant differences were found between progeny of $\mathrm{PH}$ plants and progeny of $\mathrm{NH}$ plants in \% TTC reduction and \%CMS only for G6 and G10 genotypes (Figure 2). These results indicating that the epigenetic variation is dependent on the genetic variations and it is part of the genetic variations. More than 130 epigenetic regulators were identified in Arabidopsis. Epigenetic diversity of pathways in plants is remarkable, presumably contributing to the phenotypic plasticity of plant development and the ability to survive in environments of unpredictable. Gene families encoding epigenetic modifiers can differ significantly in the number of family members between plants and mammals. (Pikaard and Scheid 2014). The prevalence of polyploidy in plants included wheat, cotton, potato, peanut, sugarcane, coffee, canola, and tobacco, suggested that it offers certain fitness advantages, such as built-in heterosis or resistance to the effects of deleterious mutations, thereby allowing duplicated genes to potentially acquire beneficial mutations, and the formation of polyploids is often associated with significant genomic and epigenetic changes (Jackson and Chen 2010). In view of the adverse effects of global climatic change, utilizing epigenetic variation for developing improved crop genotypes is of paramount importance (Singroha and 
Sharma 2019). The stability and inheritance of DNA methylation among generations has been evaluated in many different ways. Perhaps the important assessment was through the analysis DNA methylation in a mutation cumulating population in Arabidopsis thaliana (Graaf et al. 2015). Thiebaut (2019) reported in some cases, the epigenetic changes can be stably inherited to the next generation. The epigenetic variations among individuals can lead to the formation of epiallels (Kalisez and Purugganan 2004). Substantial natural variation in DNA methylation patterns exists within many plant species, and this variation can influence gene expression and plant traits (Springer and Schmitz 2017).

Dendrogram constructed based on genetic distance matrix obtained from morpho-physiological traits for progeny of heat primed (P) and non-primed $(\mathrm{N})$ genotypes under heat stress condition. Dendrogram divided all genotypes into four clusters. The first and second clusters contained G9-P, G1-P and G10-P genotypes of heat primed $(\mathrm{P})$, which each genotype given the highest genetic distance with the same genotype of non-primed under heat stress, respectively. These results indicating that the genotypes G9, G1 and G10 may be have generated the highest stress memory or epigenetic modification inherited to their progeny. Here are more evidence indicating that epigenetic process, including heritable DNA methylation, histone modification, or chromatin re-modeling independent of DNA sequence changes (Kouzarides 2007), are closely associated to the stress memory transfer to the progeny (Boyko and Kovalchuk 2011; Iwasaki and Paszkowsk، 2014). The stress memory establishment is closely associated with regulation of epigenetic (Friedrich et al. 2019). Heat shock proteins (HSPs) play a key role in conferring heat tolerance. The principle function of HSPs is to regulate protein folding and unfolding, in conjunction with their subcellular localization and the degradation of unfolded and denatured proteins (Singh et al. 2016). High temperature induces the sustained accumulation of $\mathrm{H} 3 \mathrm{~K} 4 \mathrm{me} 3$ and $\mathrm{H} 3 \mathrm{~K} 9 \mathrm{Ac}$ on, HSP22, HSP18, HSP70 and APX2 genes. Among these genes, the accumulation of H3K9Ac andH3K4me3 in HSP22, HSP18 and APX2 but not HSP70 is HSFA2 dependent (Lamke et al. 2016).

In conclusion, I evaluated heat stress tolerance induced by heat primed during the parental at both flowering and grain filling stages could be passed on the progeny of parents to more effectively respond to the successive generations under heat stress during seedling stage or grain filling in wheat. This can be exemplified by less grain yield loss per plant, higher activities of dehydrogenase enzyme, better maintenance of chlorophyll a and b content and higher cell membrane thermo-stability in the progeny of heat primed plants than in the progeny of the non-primed plants. Heat stress tolerance of transgenerational was induced by heat priming in the first generation, and this might be an effective measure to cope with severe hightemperature stresses during key growth stages in wheat production.

\section{REFRENCES}

Arnon (1949). Copper enzymes in isolated

chloroplasts. Plant Physiol. 27(1): 1-16.

Balmer A, Pastor V, Gamir J, FlorsV, Mauch-Mani B. (2015). The 'primeome': towards a holistic approach to priming. Trends Plant Sci. 20: 443-452.

Baniwal SK, Bharti K, Chan KY, Fauth M, Ganguli A, Kotak S. et al. (2004). Heat stress response in plants: a complex game with chaperones and more than twenty heat stress transcription factors. J. Biosci. 29: 471-487.

Boyko A, Blevins T, Yao Y, Golubov A, Bilichak A, Ilnytskyy Y. et al. (2010). Transgenerational Adaptation of Arabidopsis to Stress Requires DNA Methylation and the Function of Dicer-Like Proteins. PLoS ONE 5(3): e9514.

Boyko A, Kovalchuk I. (2011). Genome instability and epigenetic modification-heritable responses to environmental stress. Curr. Opin. PlantBiol. 14: 260266.

Chandler VL. (2007). Paramutation: from maize to mice. Cell .128: 641-645.

Conrath U. (2011). Molecular aspects of defence priming. Trends Plant Sci. 16: 524-531.

Conrath U, Beckers GJM, Flors V, García-Agustín P, Jakab G. et al. (2006). Priming: getting ready for battle. Mol. Plant Microbe Ineract. 19: 1062-1071.

Cossani CM, Reynolds MP. (2012). Physiological traits for improving heat tolerance in wheat, Plant Physiol. 160: 1710-1718.

Cubas P, Vincent C, Coen E. (1999). An epigenetic mutation responsible for natural variation in floral symmetry. Nature. 401: 157-161.

Driedonks N, Xu J, Peters JL, Park S, Rieu I. (2015). Multi-Level Interactions Between Heat Shock Factors, Heat Shock Proteins, and the Redox System Regulate Acclimation to Heat. Front Plant Sci. 6: 999.

Field RD, Kim D, LeGrande AN, Worden J, Kelley M. et al. (2014). Evaluating climate model performance in the tropics with retrievals of water isotopic composition from Aura TES. Geophys. Res. Lett. 41(16): 6030-6036.

Fischer RA. (1986). Physiological limitations to producing wheat in semitropical and tropical environments and possible selection criteria. Proc 
Internat Symp Wheat for More Tropical Environments, pp. 209-230. CIMMYT/UNDP, Mexico.

Fokar M, Blum A, Nguyen HT. (1998). Heat tolerance in spring wheat. II. Gram filling. Euphytica. 104: 9-15.

Friedrich T, Faivre L, Baurle I, Schubert D. (2019). Chromatin based mechanisms of temperature memory in plants.Plant Cell Environ. 42: 762-770.

Graaf V, Rate A. (2015). Spectrum, and evolutionary dynamics of spontaneous epimutations. Proc. Natl Acad. Sci. 112: 6676-6681.

Hsieh TF, Ibarra CA, Silva P, Zemach A, EshedWilliams L, Fischer RL, Zilberman D. (2009). Genome wide demethylation of Arabidopsis endosperm. Science. 324: 1451-1454.

Ibrahim AM, Quick JS. (2001). Heritability of Heat Tolerance in winter and Spring Wheat. Crop Sci. 41: 1401-1405.

Iwasaki M, Paszkowski J. (2014). Identification of genes preventing transgenerational transmission of stress-induced epigenetic states. Proc. Natl. Acad. Sci. 111, 8547-8552.

Jackson S, Chen ZJ. (2010). Genomic and expression plasticity of polyploidy. Curr Opin Plant Biol 13: 153159.

Jenner CF. (1994). Starch synthesis in the kernel of wheat under high temperature conditions. Funct. Plant Biol. 21: 791-806.

Kalisez S, Purugganan MD. (2004). Epiallele via DNA methylation: consequences for plant evaluation. Trends Ecol Waol. 19(6): 309:314.

Kouzarides T. (2007). Chromatin modifications and their function. Cell. 128: 693-705.

Kronholm I, Collins S. (2016). Epigenetic mutations can both help and hinder adaptive evolution. Mol Ecol. 25: 1856-1868.

Lamke J, Brzezinka K, Altmann S, Baurle I. (2016). A hit-and-run heat shock factor governs sustained histone methylation and transcriptional stress memory. EMBO J35: 162-175.

Larkindale J, Knight MR. (2002). Protection against heat stress-induced oxidative damage in Arabidopsis involves calcium, abscisic acid, ethylene and salicylic acid. Plant Physiol. 128: 682-695.

Latzel V, Allan E, Silveira AB, Colot V, Fischer M, Bossdorf O. (2013). Epigenetic diversity increases the productivity and stability of plant populations. Nature Commun. 4: 2875.

Mostafa GG. (2020). Genetic variability of some vegetative and flowering growth characteristics of multiflower mutant of Helianthus annuus L. plants. Scientific J. of Agricultural Sci. 2(1):1-5.
Nyquist WE. (1991). Estimation of Heritability and Prediction of Selection Response in Plant Populations. Critical Rev. in Plant Sci. 10: 235-322.

Pikaard CS, Scheid OM. (2014). Epigenetic Regulation in Plants. Cold Spring Harb Perspect Biol. 6(12): a019315.

Porter JR, Gawith M. (1999). Temperatures and the Growth and Development of Wheat A Review. Eur. J. of Agronomy. 10: 23-36.

Pradhan S, Babar Md, Bai G, Khan J, Shahi D. et al. (2020). Genetic dissection of heat-responsive physiological traits to improve adaptation and increase yield potential in soft winter wheat. BMC Genomics. 21: 315 .

Prasad PVV, Staggenborg SA, Ristic Z. (2018). Impacts of drought and/or heat stress on physiological, developmental, growth, and yield processes of crop plants, in: L.R. Ahuja, V.R. Reddy, S.A. Saseendran, Q. Yu (Eds.), Response of Crops to Limited Water: Understanding and Modeling Water Stress Effects on Plant Growth Processes, Advances in Agricultural Systems Modeling 1: Transdisciplinary Research, Synthesis, and 38 THE CROP JOURNAL 6 (2018) 32 - 41 Applications, American Society of Agronomy, Crop Science Society of America, Soil Science Society of America, Madison, WI, USA 2008: pp. 301-355.

Rasmann S, Vos MD, Casteel CL, Tian D, Halitschke R. et al. (2012). Herbivory in the previous generation primes plants for enhanced insect resistance. Plant Physiol. 158: 854-863.

Ristic Z, Bukovnik U, Momčilović I, Fu JM, Prasad PVV. (2008). Heat-induced accumulation of chloroplast protein synthesis elongation factor, EF-Tu, in winter wheat. Aust. J. Plant Physiol. 165: 192-202.

Ristic Z, Bukovnik U, Prasad PVV. (2007). Correlation between heat stability of thylakoid membranes and loss of chlorophyll in winter wheat under heat stress, Crop Sci. 47: 2067-2073.

Ritchier SW, Nguyen HT, Holaday AS. (1990). Leaf water content and gas-exchange parameters of two wheat genotypes differing in drought resistance. Crop Sci. 30: 105-111.

Shah NH, Paulsen GM. (2003). Interaction of drought and high temperature on photosynthesis and grainfilling of wheat. Plant Soil. 257: 219-226.

Singh RK, Jaishankar J, Muthamilarasan M, Shweta S, Dangi A, Prasad M. (2016). Genome-wide analysis of heat shock proteins in $\mathrm{C} 4$ model, foxtail millet identifies potential candidates for crop improvement under abiotic stress. Sci Rep. 6: 32641.

Singroha G, Sharma B. (2019). Epigenetics modification in plant under abiotic stress. Open access 
peer-reviewed:chapter.

Doi:10.5772/interchopen.84455.

Springer NM, Schmitz RJ. (2017). Exploiting induced and natural epigenetic variation for crop improvement Nature Rev. Genetics. 18: 563-575.

Thiebaut I, A Hemerly AS, Ferreira PCG. (2019). A Role for Epigenetic Regulation in the Adaptation and Stress Responses of Non-model Plants. Front Plant Sci. 10: 246.

Verhoeven KJF, Vonholdt BM, Sork VL. (2016). Epigenetics in ecology and evolution: what we know and what we need to know. Mol. Ecol. 25: 16311638 .

Vogt G. (2018). Investigating the genetic and epigenetic basis of big biological questions with the parthenogenetic marbled crayfish: A review and perspectives. J Biosci. Mar. 43(1): 189-223.

Wang X, Cai J, Jiang D, Liu F, Dai T, Cao W. (2011). Pre anthesis high-temperature acclimation alleviates damage to the flag leaf caused by post-anthesis heat stress in wheat. J. Plant Physiol. 168: 585-593.
Wang X, Cai J, Liu F, Jin M, Yu H. et al. (2012). Preanthesis high temperature acclimation alleviates the negative effects of post-anthesis heat stress on stem stored carbohydrates remobilization and grain starch accumulation in wheat. J. Cereal Sci. 55: 331-336.

Wang X, Cai J, Liu F, Dai T, Cao W, Wollenweber B, Jiang D. (2014). Multiple heat priming enhances thermo-tolerance to a later high temperature stress via improving subcellular antioxidant activities in wheat seedlings. Plant Physiol. Biochem. 74: 185-192.

Wang X, Xin C, Cai J, Zhou G, Dai T, Cao W, Jiang D. (2016). Heat Priming Induces Trans-generational Tolerance to High Temperature Stress in Wheat. Frontiers in Plant Sci. 7: 501.

Yang X, Tian Z, Sun L, Chen B, Tubiello FN, Xu Y. (2017). The impacts of increased heat stress events on wheat yield under climate change in China, Clim. Chang. 140: 605.

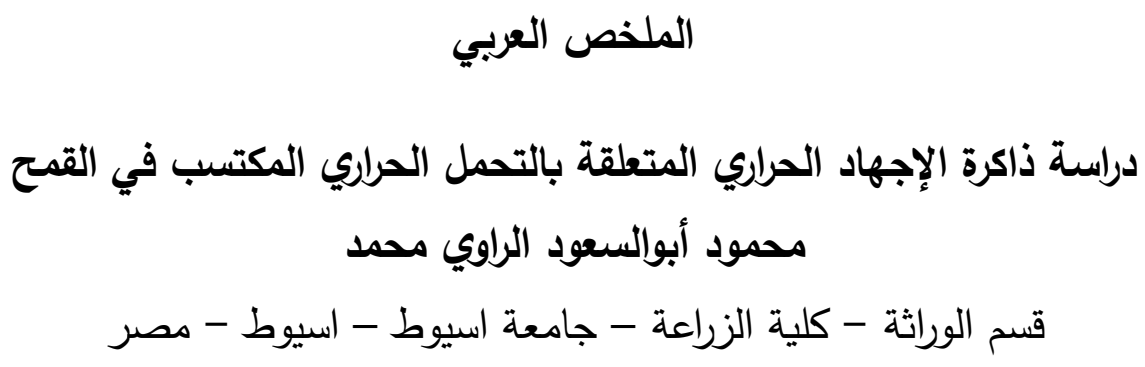

يعتبر القهح من أهم النباتات التي تعاني بشكل متكرر من آثار الإجهاد الحراري. في هذه الدراسة، تم دراسة المعاملة الحرارية لجيل الآباء على تحمل الحرارة لنسل هذه الآباء في الأجيال المتعاقبة في مرحلتي الشتلات والنضج. أثناء مرحلة الشتلات وتحت درجات الألات الحرارة المرتفعة ، أظهرت

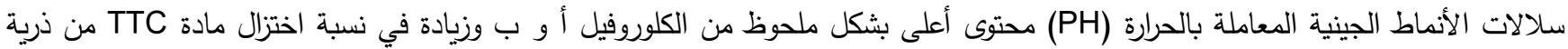
الأنماط الجينية غير معاملة بالحرارة (NH). في مرحلة النضج ، تم الحصول على محصول حبوب أعلى للنبات بالنسبة للطرز الجينية المعاملة بالحرارة مقارنة بالأنماط الوراثية الغير معاملة بالحرارة تحت ظرئ بارئ الإجهاد الحراري، والتي ارتبطت بشكل أساسي بارتفاع وزن الألف الحبة للأنماط

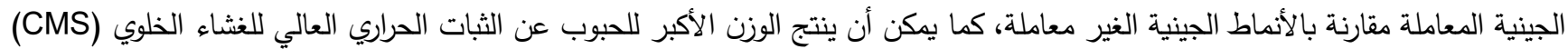

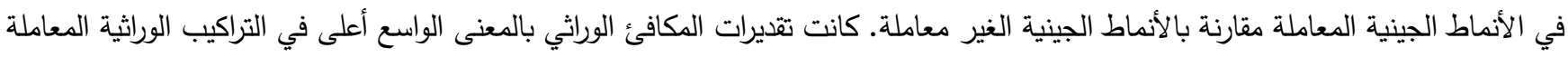
مقارنة بالطرز الوراثية الغير معاملة لدعظم الصفات المدروسة في مرحلتي الشتلات والنضج. لقد اظهرت مصفوفة المسافة الوراثية التي تم الحصول

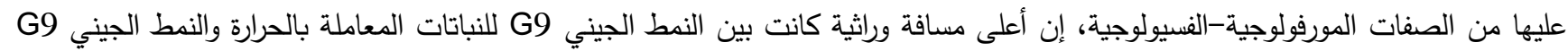

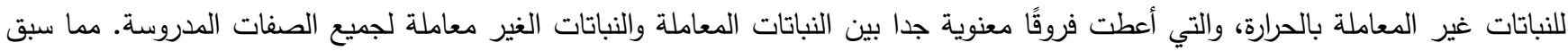

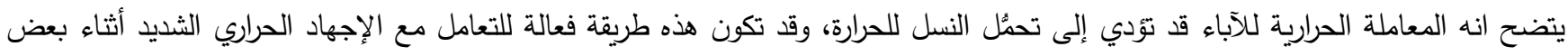
مراحل النمو المختلفة في القهح. 